\title{
La cultura del progetto, quando è design Introduzione
}

\author{
Michela Deni \\ PROJEKT (EA 7447) Université de Nîmes, FR \\ michela.deni@unimes.fr \\ $<$ https://projekt.unimes.fr/membres/michela-deni/>
}

\begin{abstract}
When is Design, we find those characteristics and values of "design culture" that regard design processes and methods, designers' skills and attitudes. When these necessary preconditions are fulfilled, in many cases it is design. In some other situations, it is just a matter of finding a strategy to light up night dinners under the starry sky. In the Introduction of Ocula 24 we investigate the conditions that favour the emergence of design processes typical of design and of its evolution, up to social design which, by overcoming incremental and inertial innovations, drives radical social innovations.
\end{abstract}

\section{Keywords}

Semiotics of design project; Design; Design Culture; Social design.

\section{Contents}

Introduzione

1. La cultura del progetto

2. Dal designer al design diffuso

3. Quando è design, la cultura del progetto: una condizione necessaria ma non sufficiente

Conclusioni

Bibliografia 
Vol 21, No 24 (October 2020) • DOI: 10.12977/ocula2020-39

Michela Deni | Introduzione. La cultura del progetto, quando è design

\section{Introduzione}

Quando è design è il titolo di un convegno che, per iniziativa di Paolo Fabbri, si è svolto nel Centro Internazionale di Semiotica dell'Università di Urbino nel settembre del 2018, e che abbiamo organizzato insieme a Dario Mangano con la collaborazione di Projekt (EA 7447), Laboratorio di ricerca in innovazione sociale attraverso il design dell'Università di Nîmes (Francia). Gli articoli presenti in questo numero di Ocula sono il risultato dell'evoluzione delle riflessioni della maggioranza dei relatori presenti al convegno anche in seguito agli scambi iniziati proprio in quell'occasione. Abbiamo deciso di non pubblicare gli atti del convegno, ma un numero tematico con articoli originali valutati in peer review.

Quando è design è dunque un titolo affermativo, è la risposta plurale di studiosi e professionisti che lavorano nel settore del design e che si sono interrogati in molti modi sulle condizioni che generano design. Non si tratta quindi di una riflessione ontologica ma, come ha suggerito Paolo Fabbri proponendo di partire da Goodman (1968) e parafrasandolo, si tratta piuttosto di una riflessione sui processi che portano al design, nelle sue differenti declinazioni. Il design come risultato materiale e immateriale di un approccio culturale, sociale, antropologico, metodologico e semiotico che è proprio alla cultura del progetto. Ci torneremo tra poco.

La riflessione sul "quando" è design ci accompagna da tempo. Nel settore del design industriale la definizione di design ha coinciso lungamente con il risultato del lavoro dei designer che si manifesta in prodotti concreti (materiali e immateriali) come oggetti d'uso, spazi, comunicazione, servizi ecc. In quest'ambito la definizione della World Design Organisation è esaustiva:

Industrial Design is a strategic problem-solving process that drives innovation, builds business success, and leads to a better quality of life through innovative products, systems, services, and experiences. Industrial Design bridges the gap between what is and what's possible. It is a trans-disciplinary profession that harnesses creativity to resolve problems and co-create solutions [...] Industrial Design provides a more optimistic way of looking at the future by reframing problems as opportunities. [...] Industrial Designers place the human in the centre of the process. They acquire a deep understanding of user needs through empathy and apply a pragmatic, user-centric problem-solving process to design products, systems, services, and experiences. ${ }^{1}$

A partire da diverse definizioni di design condivise in gran parte da professionisti e studiosi del settore, il design è indicato come il risultato di un processo progettuale, industrialmente trasponibile e replicabile, quasi sempre autoriale o imprenditoriale, poco importa se individuale o collettivo, comunque riconosciuto o riconoscibile (cfr. Alessi 2016).

1 <http://wdo.org/about/definition/> (pagina consultata il 10/o9/20). 
Vol 21, No 24 (October 2020) • DOI: 10.12977/ocula2020-39

Michela Deni | Introduzione. La cultura del progetto, quando è design

Nell'ultimo decennio il termine "design" si è esteso a numerosi settori, ${ }^{2}$ tra questi uno in particolare è quello del social design e del design per l'innovazione sociale. ${ }^{3} \mathrm{Il}$ social design è importante anche perché mette alla prova ogni tentativo di definizione del (e dei) design evidenziando come il cercare di rispondere alla domanda di "cosa è design" non sia pertinente poiché il denominatore comune a certi settori è appunto il processo, non necessariamente il risultato: ciò accade ad esempio nel design delle politiche pubbliche, in settori come le amministrazioni, l'insegnamento, la sanità. ${ }^{4}$ Il processo in questi casi è inteso come il modo di affrontare inizialmente l'analisi delle pratiche quotidiane (o specifiche) di tutti i soggetti coinvolti per identificare strategie progettuali finalizzate a dare una risposta concreta a un problema o a una mancanza che riguarda ampie categorie di persone (l'accesso, l'accompagnamento, l'informazione, la gestione ecc.). Come sostiene Ezio Manzini (2018: 114) «una politica del quotidiano è l'effetto sistemico di un progetto di vita»e, aggiungiamo, va quindi affrontata con metodi sia complessi che complessivi (pluridisciplinari) che permettano una comprensione globale e una risoluzione che si avvale di numerose competenze.

Capita allora sempre più spesso di chiedersi "quando" è design, in particolare di fronte ad alcuni progetti commissionati da istituzioni, ospedali e collettività. E ce lo si chiede soprattutto quando è difficile identificare "il design" come risultato: in questi casi il design è nel processo progettuale, vale a dire nella modalità attraverso cui si è giunti a una soluzione grazie alla postura critica, analitica e pragmatica di un'équipe di designer che trasforma un problema in opportunità. ${ }^{5}$ In tali occasioni (progettuali, pedagogiche, di consulenza)

2 Industrial design, graphic design, urban design, interaction design, fashion design, food design, interior design, car design, digital design, communication design, service design, ecc.

3 Una spiegazione semplice e immediata sul social design e sul design per l'innovazione sociale si trova in questo breve video realizzato dall'agenzia francese La Bobine (<www.labobine.co>): <https://www.youtube.com/watch?v=LjuqoqEpIR8\& feature $=$ emb_title $>$; (pagina consultata il 10/09/20).

4 Tra molti altri ne indichiamo alcuni nella realtà francese: le iniziative de La $27 e$ Région, laboratorio per trasformare le politiche pubbliche (http://www. la27eregion.fr); CALME (Comme À La Maison en EHPAD), un progetto per le case di riposo (<http://projetcalme.fr >); La Ressource de l'autonomie, un progetto per migliorare l'autonomia delle persone anziane a domicilio e presentato in questo numero di Ocula da M. Royer (<http://ressource-autonomie.fr $>$ ); CancerAdom, Come migliorare l'esperienza della malattia a domicilio (<http://canceradom.fr $>$ ); il Labah, Laboratorio di accoglienza e ospitalità del GHU di Parigi, centro ospedaliero di psichiatria e neuroscienze (<http://www.ght-paris.com/fr/le-labah/>); La Fabrique de l'hospitalité, laboratorio d'innovazione degli ospedali universitari di Strasburgo (<http://www.lafabriquedelhospitalite.org >).

5 Altri esempi interessanti si trovano tra i progetti degli studenti della laurea magistrale Master DIS (Design Innovation Société) dell'Università di Nîmes: si tratta di progetti di social design commissionati dalle regioni (Occitanie, PACA), da ospedali (CHU Marseille, CHU Nîmes), dalla Caf, dal CROUS, dalla provincia (Conseil départemental du Gard), ecc. I progetti si trovano sul sito della laurea magistrale, in particolare a questo indirizzo: <https://dis.unimes.fr/projets/>_(pagina consultata il 10/09/20). 
Vol 21, No 24 (October 2020) • DOI: 10.12977/ocula2020-39

Michela Deni | Introduzione. La cultura del progetto, quando è design

se si è abituati ad avere il ruolo di chi analizza il contesto in cui si interviene per accompagnare e strutturare le fasi progettuali, è facile disorientarsi perché si è di fronte a designer che hanno integrato a modo loro tutte le competenze accessorie e anche di più. ${ }^{6}$ Da semiotici però è inevitabile cercare la sistematicità con inguaribile ottimismo e domandarsi dove e quali siano le ricorrenze di quel "tutto" (ma quale tutto?) "che si tiene". Cosa identificare allora, cosa legittimare e valorizzare del processo progettuale, all'interno ma anche agli occhi dei committenti? Il risultato? I metodi progettuali? Il processo?

Se di processo si tratta, abbiamo deciso di ricorrere alla cultura del progetto, un concetto che, in particolare in Italia, diamo per scontato poiché, prima di formarsi in scuole di design che hanno tardato ad arrivare, i designer erano in particolare architetti e ingegneri quindi possiamo affermare che il design è nato, vissuto e evolve sempre in questa cultura.

Cercheremo di spingerci oltre l'implicito per capire meglio a cosa ci si riferisce quando si parla di "cultura del progetto" e come tale cultura evolva nel tempo.

\section{La cultura del progetto}

Per descrivere cosa si intenda per cultura del progetto, in generale possiamo dire che si tratta di una serie di valori, sensibilità, competenze e principi progettuali maturati e trasmessi nel tempo, in vari modi.7 Trasmessi implicitamente dal tessuto culturale e sociale in cui si vive, dal territorio che si abita, dalle istituzioni culturali e, esplicitamente, dalle istituzioni delegate alla didattica del design.

Ispirandoci in particolare agli scritti, ai progetti e alle iniziative di Adriano Olivetti, Bruno Munari, Tomás Maldonado, Ezio Manzini e Paolo Deganello, possiamo iniziare a definire la cultura del progetto descrivendola con alcune specificità caratterizzanti: 1) Metodi progettuali; 2) Interdisciplinarietà e competenze trasversali; 3) Creatività; 4) Integrazione dell'essere umano. Per quanto riguarda l'ultimo punto, quello della presa in conto dell'utente nel progetto (user-centered design), sebbene storicamente si tratti di una delle ragioni della nascita del design, negli ultimi anni questo aspetto si è incarnato con il social design e il co-design dove «le persone non sono viste solo come portatrici di bisogni, ma anche di capacità (non solo come parte dei problemi che si trovano ad affrontare, ma anche come attori della loro soluzione)» (Manzini 2018: 75).

Oltre alle specificità indicate, ci sono alcuni valori di base che pertengono all'origine e all'affermazione nel tempo della cultura del progetto: a-Etica; b-Sostenibilità; c) Qualità della vita (praticità, estetica, identità). Come vedremo in conclusione, tali valori si sono naturalmente evoluti nel tempo.

6 Semiotiche, etnografiche, sociologiche, economiche, gestionali, architettoniche, artistiche, ecc. Per questo anche la formazione dei designer del XXI secolo, secondo Donald Norman, deve tenere conto di tale evoluzione del design e dei modi di praticarlo: <https://www.youtube.com/watch?v=7FJNsqoC4tI >.

7 Cfr. Julier e Munch (2019). 
Vol 21, No 24 (October 2020) • DOI: 10.12977/ocula2020-39

Michela Deni | Introduzione. La cultura del progetto, quando è design

\subsection{Il design come metodo progettuale}

La prima specificità che abbiamo indicato per definire la cultura del progetto è quella dei metodi progettuali a cui dedichiamo un paragrafo, al contrario delle altre specificità indicate che abbiamo già discusso altrove, interrogandoci sulla creatività (cfr. Deni 2016); sull'integrazione dell'utente nei progetti (cfr. Deni 2018; Deni e Catoir-Brisson 2019; Deni 2020) e sulle competenze sia professionali che teoriche mobilitate dai designer (cfr. Deni 2019).

I metodi progettuali legati alla cultura del progetto permettono la gestione di quella che Bruno Munari chiama la creatività in contrapposizione alla creazione $^{8}$ la quale, per Munari, si basa solo sulla fantasia e riguarda l'arte e le arti applicate, settori che secondo lui sono molto diversi dal design. La creatività «è una capacità produttiva dove fantasia e ragione sono collegate per cui il risultato che si ottiene è sempre realizzabile praticamente» (1971: 87) e, tale risultato, per Munari viene compreso dalle persone che possono allora appropriarsene. In questi termini: «Il metodo progettuale non è altro che una serie di operazioni necessarie, disposte da un ordine logico dettato dall'esperienza. [...] Creatività non vuol dire improvvisazione senza metodo» (1981: 16-17). Continua con maggior precisione Munari: «La serie di operazioni del metodo progettuale è fatta di valori oggettivi che diventano strumenti operativi nelle mani di progettisti creativi» (1981: 17).

Per Munari l'opposizione tra design e arte applicata riguarda sostanzialmente l'approccio al processo progettuale: la creazione è propria al lavoro di un artista, il cui prodotto è un'opera quasi sempre individuale e autoriale; la progettazione è propria al lavoro creativo del designer che opera basandosi su metodi razionali. C'è quindi una forte opposizione tra "artista e designer" che, non a caso, è anche il titolo di un libro di Munari (1971). Tale opposizione è importante per il tema di questo numero di Ocula poiché non si tratta di definire il design appunto, ma di stabilire come e quando ci sono le condizioni proprie e propizie all'emergere del design. Queste condizioni dipendono in gran parte dall'approccio metodologico. In modo certamente semplificativo ma esplicito per chiarire la propria posizione, Munari identifica un "senza metodo" dell'artista opposto a "con metodo" caratteristico dei processi progettuali del designer. Dove per "metodo" si intende una modalità di agire (il come) che presuppone una strategia dispiegata temporalmente (il quando) nella sequenzialità delle operazioni caratterizzate dalla consapevolezza e dalla capacità di analisi del designer sulla propria pratica. Inoltre, secondo Munari l'artista crea soggettivamente affermando il proprio stile, mentre il designer basa il proprio metodo progettuale sulla ricerca e sulla conoscenza di altri metodi facendo uno studio attento di ciò che è stato fatto in precedenza. Tale consapevolezza e tali capacità analitiche permettono al designer di rispondere specificamente a ogni problema che gli si pone (dal design industriale al social design).

8 Cfr. Munari 1971 e 1981; Deni 2016. 
Vol 21, No 24 (October 2020) • DOI: 10.12977/ocula2020-39

Michela Deni | Introduzione. La cultura del progetto, quando è design

Il vero designer può progettare un mobile, un giocattolo, una struttura metallica, può occuparsi di un problema di illuminazione o altro, tutti diversi tra loro, non perché sia un genio, ma perché ha un metodo di progettazione che lo conduce a soluzioni logiche ed anche estetiche tutte diverse, secondo i materiali, le tecniche e le funzioni. (1971: 51-52)

Per Munari (1971) è quindi un errore rivolgersi al designer una volta che la progettazione sia già avanzata e, già negli anni Sessanta, sottolinea un "grande malinteso" (in particolare in paesi come la Francia): la mancanza di distinzione tra il design delle Arti Applicate e il design "vero e proprio" (progettuale, diremmo noi). Il design delle Arti Applicate consiste in un effetto di superficie e riguarda l'estetica (abbellire un oggetto per esempio); il design che viene dall'integrazione di architettura, ingegneria, arte e industria riguarda invece la visione d'insieme di un progetto (pratica d'uso, contesto, ergonomia, materiali, valori, ecc.).

La conoscenza complessiva e la consapevolezza sono allora le condizioni essenziali del design, come ricorda con forza Tomás Maldonado, "intellettuale politecnico", 9 già nel 1970 ne La speranza progettuale: un'esortazione lungimirante e ancora attuale - persino urgente - rivolta ai progettisti affinché assumessero attivamente il loro ruolo politico, sociale e culturale attraverso una progettazione sostenibile ${ }^{10}$ per assicurare un "ambiente artefatto" adeguato evitando la catastrofe ambientale. La progettazione è allora la gestione di tutti questi aspetti, dove la «gestione è il comportamento conoscitivo ed operativo tramite il quale si trasforma l'informazione in azione» (Madonado 1970: 101). Per sottolineare l'importanza di operare attraverso metodi progettuali Tomás Madonando sottolinea la propria fiducia nella «funzione rivoluzionaria della razionalità applicata» (cfr. Madonado 1970).

Il metodo è dunque un aspetto caratterizzante e identificativo della cultura del progetto, dal design industriale al social design, passando per tutti i settori del design (grafica, prodotto, spazio ecc.). Il designer a cui si riferiscono Munari, Maldonado e Manzini non decide quale sarà il proprio progetto ma si concentra su un aspetto significativo che per commissione o per osservazione personale è chiamato a risolvere o a trasformare in una soluzione preferibile. Come afferma Herbert A. Simon: «Everyone designs who devises courses of action aimed at changing existing situations into preferred ones» (Simon 1969: 111).

Non soltanto i teorici e i maestri del design ci aiutano a comprendere cosa si intenda per cultura del progetto, ma possiamo osservare anche industrie storiche che la cultura del progetto l'hanno concretamente "incarnata". ${ }^{11} \mathrm{~L}$ 'Olivetti è stata un'azienda esemplare in questo senso, soprattutto tra il 1932 e il 1960 introducendo il design come settore centrale della propria politica

9 Dal titolo del libro di Giovanni Anceschi dedicato a Tomás Maldonado (2020).

10 Negli stessi anni Victor Papanek condivideva le stesse preoccupazioni sulla sostenibilità di un design per il "mondo reale" (1971).

11 Cfr. l’intervista a L. Gallino (Ceri ed. 2014). 
Vol 21, No 24 (October 2020) • DOI: 10.12977/ocula2020-39

Michela Deni | Introduzione. La cultura del progetto, quando è design

di impresa (in particolare design di prodotto, grafica e architettura) durante la direzione di Adriano Olivetti. Grazie alla centralità del design e ai metodi progettuali, l'Olivetti produceva con successo prodotti innovativi, di grande qualità estetica e funzionale, innescando un circolo virtuoso che consentiva di reinvestire il fatturato in ricerca e sviluppo permettendo all'impresa di migliorare ancora. Nel 1933, quando il nazismo chiuse il Bauhaus, Adriano Olivetti invitò come collaboratore uno dei migliori designer della scuola, Xanti Schawinsky. In azienda il design integrava il processo progettuale dall'inizio e agli ingegneri vennero affiancati designer come Ettore Sottsass. ${ }^{12}$ All'Olivetti, i designer condizionavano e guidavano molte scelte, come quelle legate alla politica culturale e sociale d'avanguardia per il benessere dei lavoratori, una politica molto simile al welfare aziendale attualmente più avanzato (organizzazione del lavoro, qualità dello spazio architettonico, servizi culturali, educativi e ricreativi, sanitari, ecc.). Ciò accadde molti anni prima che le aziende diventassero definitivamente soggetti riconoscibili non soltanto grazie ai loro prodotti ma anche per la propria politica di responsabilità sociale d'impresa basata su valori incarnati in azioni concrete. ${ }^{13}$

\subsection{Il designer: attitudine progettuale}

È venuto il momento di chiedersi se "il designer" a cui facciamo riferimento sia una figura professionale identificabile grazie a un'attitudine particolare che ne determina l'approccio al progetto.

Ancora una volta ricorriamo a Bruno Munari per capire come operi un designer: «È un progettista con senso estetico" (1966: 25), non ha necessariamente uno stile personale poiché la sua unica preoccupazione è di arrivare «alla soluzione del progetto secondo quegli elementi che l'oggetto stesso, la sua destinazione ecc. suggeriscono» (1966: 44). A differenza dell'artista, scrive ancora Munari, «Il designer oggettivo ha come meta l'estetica come una tecnica pura» (1971: 10) poiché «l'estetica del designer è più vicina a quella di un ingegnere» (1971: 180).

È importante tuttavia ricordare che Munari si riferisce al design industriale precedente agli anni Novanta periodo che, come accade anche per la moda, ha dato origine a un effetto di personalizzazione del prodotto e della marca. Quando Munari evoca l'"estetica" intende la "coerenza formale, qualcosa di simile a ciò che vediamo in natura [...] Il designer cerca di costruire l'oggetto con la stessa naturalezza con la quale in natura si formano le cose [...] aiuta l'oggetto a formarsi con i suoi propri mezzi» (1966: 26-27), poiché, insiste, bisogna «dare a ogni cosa la sua logica struttura, la sua logica materia e di conseguenza la sua logica forma [...] coerente all'uso [...] in rapporto alle sue pos-

12 Per un approfondimento sulla centralità del design all'Olivettivedi anche: $<$ https:// web.archive.org/web/20080429190509/http://www.storiaolivetti.telecomitalia.it/cgibin/Societa/design_olivetti.asp> (pagina consultata l'11/o9/2020).

13 In questo senso l'Olivetti anticipa anche ciò che si indica come "forma marca" (cfr. Semprini 2005). 
Vol 21, No 24 (October 2020) • DOI: 10.12977/ocula2020-39

Michela Deni | Introduzione. La cultura del progetto, quando è design

sibilità costruttive» (1966: 31), fino ad aggiungere, nella sua ricerca costante dell'equilibrio, che «Il 'bello' è la conseguenza del 'giusto'. Una progettazione esatta dà un oggetto bello» (1966: 31).

$\mathrm{Si}$ consideri il periodo storico in cui Munari - designer Moderno, e "scientista", come lo definisce Paolo Deganello (2019: 18) - opera e scrive valorizzando strategie razionali del progetto che si rispecchiano nell'estetica. Un'estetica che non ha a che fare con la "bellezza" ma con la regola della "natura" e con la qualità della vita. In altri termini si tratta di "pensare giusto", come direbbe Alain Findeli (cfr. 2005), un pensiero "in azione" e nel contesto pragmatico delle questioni che si è chiamati a risolvere.

Gli oggetti "naturali", indicati da Munari come esempi da prendere a modello per comprendere i fondamenti della progettazione, integrano funzionalità, praticità, estetica, sostenibilità. Tra altri, il celeberrimo esempio dell'arancia che riassume le qualità necessarie a ogni progetto riuscito poiché è costituito

da una serie di contenitori modulati a forma di spicchio, disposti circolarmente attorno un asse centrale verticale, al quale ogni spicchio appoggia il suo lato rettilineo mentre tutti i lati curvi, volti verso l'esterno, danno, nell'insieme, una forma globale, una specie di sfera. L’insieme di questi spicchi è racchiuso in un imballaggio [...] duro alla superficie esterna e rivestito da un'imbottitura morbida interna, di protezione [...] Ogni contenitore è a sua volta formato da una pellicola plastica [...] manovrabile nella scomposizione della forma globale. Ogni spicchio è tenuto insieme da un piccolo adesivo. L’imballaggio [...] si può gettare. Ogni spicchio ha esattamente la forma della disposizione dei denti nella bocca umana (...) Di solito gli spicchi contengono [...] un piccolo seme [...] un piccolo omaggio che la produzione offre al consumatore nel caso che questi volesse avere una produzione personale [...] Da notare il disinteresse economico [...] L'arancia è un oggetto quasi perfetto dove si riscontra l'assoluta coerenza tra forma, funzione, consumo [...] Unica concessione decorativa [...] la ricerca 'materica' della superficie dell'imballaggio [...] Forse per ricordare la polpa interna dei contenitori. Comunque, un minimo di decorazione, perfettamente giustificato, bisogna riconoscere. (Munari 1966: 136-137)

L'acidità del succo dell'arancia permette inoltre a chi la consuma di disinfettarsi le mani prima di mangiarne uno spicchio.

Per Munari i "piselli" sono un altro esempio utile per "capire la natura": sono pillole alimentari con un "astuccio elegante" e semplice da aprire.

L'osservazione della natura permette allora di capire la logica progettuale, secondo Munari, nell'economia del rispetto della pratica d'azione, del materiale e della sua relazione con l'ambiente, della forma, della decorazione e, infine, della relazione necessaria tra le componenti. In altri termini, si tratta delle caratteristiche e dei valori che abbiamo individuato nella cultura del progetto (metodi progettuali, competenze trasversali, creatività e integrazione dell'essere umano tramite la previsione della pratica d'azione; poi etica, sostenibilità e qualità della vita che coniuga la praticità con l'estetica). 
Vol 21, No 24 (October 2020) • DOI: 10.12977/ocula2020-39

Michela Deni | Introduzione. La cultura del progetto, quando è design

\section{Dal designer al design diffuso}

La cultura del progetto, specificità che nel campo del design inizialmente si è espressa nel design industriale, si è progressivamente spostata verso settori più ampi. Chiara Alessi (2016) indica una svolta negli anni '10, periodo in cui c'è il Design senza designer poiché, in qualche modo, si perde l'importanza del "chi" fa design. Tale processo si manifesta attraverso una cultura diffusa «dell'accessibilità [...] agli strumenti di progettazione e creazione» (Alessi 2016: VIII) grazie all'open source e ai FabLab. Ciò comporta un anonimato ritrovato, un ripensamento dell'autorialità e la nascita di nuove collaborazioni per progetti sempre più collettivi che emergono grazie a competenze $\mathrm{e}$ passioni diverse, come accade con i pro-am (da professional and amateur). C'è anche il superamento della mediazione operata dalla distribuzione poiché chi progetta e produce spesso è anche chi "usa": queste figure che si sovrappongono sono sintetizzate col termine produser (produttore e utente). Tali fenomeni emergenti vengono assorbiti dal sistema anche a livello industriale, come accade con Ikea Hacking (Alessi 2016: XV) in cui sono gli stessi clienti a valorizzare e suggerire la personalizzazione dei prodotti di questa marca. Si arriva inoltre «a una trasfusione dell'intelligenza del design dal prodotto in sé ai processi che lo regolano» (Alessi 2016: XX), in altri termini si arriva a una diffusione della cultura del progetto fino al Design, When Everybody Designs di Ezio Manzini in cui il design detiene ancora un ruolo centrale per stimolare e rispondere ai bisogni, ai processi progettuali e creativi che nascono dal basso.

Il designer che favorisce questi processi non assomiglia all'ingegnere di Bruno Munari né a quello di Lévi-Strauss (1962), al contrario è un designer con le qualità del bricoleur della nota opposizione ripresa anche da Floch (1995). Il progettista-ingegnere parte, secondo Manzini, da un obiettivo preciso definendo i mezzi per raggiungerlo; quando invece il progettista-bricoleur

opera in base a un'intenzionalità, ma [...] approssima i suoi risultati assemblando oggetti preesistenti, che egli individua, decontestualizza e reinterpreta modificandone il significato e alcuni dettagli [...] gli oggetti del suo magazzino (raccolti precedentemente) hanno una forma, legata alla loro precedente funzione, incontrandoli il progettista bricoleur può riconoscervi una nuova forte potenzialità funzionale o espressiva, decidere di dar loro un particolare ruolo e cambiare di conseguenza il suo obiettivo finale. (Manzini 2018: 78-79)

Si tratta quindi di «un progetto dialogico, capace di ascoltare con attenzione la realtà e, nel caso, riorientare il proprio percorso» (Manzini, 2018: 79). Il miglioramento della qualità della vita e dell'abitabilità del mondo (cfr. Findeli 2005) deve favorire un'innovazione sociale su più larga scala. Le qualità determinanti per la competenza progettuale descritta da Manzini (2018) sono: il senso critico (cosa non ci va bene nella realtà), la creatività (ci permette di immaginare come potrebbe essere), la capacità di analisi (vincoli, risorse disponibili e opportunità) e il senso pratico (per mettere in atto le strategie 
Vol 21, No 24 (October 2020) • DOI: 10.12977/ocula2020-39

Michela Deni | Introduzione. La cultura del progetto, quando è design

di azione). Progettare non significa quindi stabilire a priori quale progetto si farà (ad esempio una lampada), significa invece analizzare la realtà e chiedersi come migliorarla facendo una sintesi tra i dati ricavati (ad esempio la mancanza di illuminazione intima e a basso impatto). Il progettista-bricoleur descritto da Manzini adatterà ciò che lo circonda per trovare una soluzione inedita attraverso la propria creatività.

\section{Quando è design, la cultura del progetto: una condizione necessaria ma non sufficiente}

Abbiamo ripercorso fino a questo momento le caratteristiche e i valori della cultura del progetto che riguardano i processi e i metodi progettuali, le attitudini dei designer e il design diffuso. Quando ci sono queste condizioni necessarie, in molti casi è design.

Tuttavia, tali condizioni non ci permettono ancora una risposta definitiva.

\subsection{L'illuminazione nel cielo stellato}

Concentriamoci su un esempio che riguarda una situazione specifica, certamente non esaustiva ma, a nostro avviso, significativa: pensiamo a una situazione di vacanza in una piccola isola in cui l'elettricità è arrivata da una trentina d'anni, solo nelle abitazioni e non nelle strade. Tale scelta oramai non dipende più soltanto dalla difficoltà tecnico-logistica di estendere l'illuminazione all'unica strada che percorre l'isola, è una scelta pensata anche in virtù di preservare il cielo stellato dall'inquinamento luminoso. Una scelta forse discutibile da un punto di vista "funzionale" ma più che condivisibile se si confrontano le necessità dell'uso della strada illuminata dai fari dei pochi veicoli nelle ore notturne ampiamente ripagate dal contatto con la natura immersa nella volta celeste. In questa piccola isola in estate si cena all'aperto, nel patio della casa, quasi sempre a lume di candela. Negli anni le tavole sono state illuminate da lampade a olio, ultimamente da elettricità o lampade a energia solare, ma in gran parte si sono sempre usate le candele: per l'atmosfera, per un omaggio al passato e agli elementi naturali. Ecco che una questione come un'altra da risolvere, per i commensali così come per un designer, è quella dell'illuminazione della tavola. Non si tratta quindi di decidere di progettare una nuova lampada per illuminare ma, se ragioniamo come un designer, si tratta di chiedersi se siamo di fronte a un problema di illuminazione che privilegi l'atmosfera e l'intimità della condivisione della tavola. La necessità quindi sarà quella di un punto luce che non abbia un impatto eccessivo sui generatori elettrici e non produca inquinamento luminoso diffuso.

Coloro che hanno integrato una certa cultura del progetto prendono tempo per definire il problema e non si fermano sulla prima soluzione evidente (cfr. Munari 1981). Nel contesto descritto sarà più utile progettare un sistema di illuminazione utilizzando, come soluzione ottimale, i materiali e gli oggetti a disposizione soprattutto se da riciclare. Aggiungiamo anche che l'acqua del rubinetto dell'isola non è potabile, si deve perlopiù acquistare in bottiglia poi- 
Vol 21, No 24 (October 2020) • DOI: 10.12977/ocula2020-39

Michela Deni | Introduzione. La cultura del progetto, quando è design

ché anche l'acqua piovana non è sufficiente ai bisogni legati alla vita contemporanea e domanda un lavoro accurato e costante di pulizia del pozzo. Ecco che, riunite queste informazioni, magari proprio durante un pasto, può venire in mente una soluzione creativa e a portata di mano, dunque adatta, economica e ecologica: per progettare una fonte luminosa discreta, che crei atmosfera e intimità attorno al tavolo all'aperto di una casa su una piccola isola marina, sarà necessaria almeno una candela protetta dal vento, dotata di un paralume che non ingombri troppo il tavolo e ancora meglio che non si debba acquistare perché sull'isola non c'è produzione e si trovano quasi solo oggetti essenziali che arrivano dalla terraferma. Tenendo conto di tutte le condizioni indicate, il problema di un'illuminazione intima e non appariscente può essere risolto "puntualmente" con una bottiglia di plastica da riciclare: si taglia in due; la metà inferiore viene stabilizzata con una piccola quantità d'acqua; la parte superiore viene capovolta e inserita nell'altra metà; si scioglie un po' di cera per incollare una candela al tappo che ne diverrà la base; la candela sarà protetta dalla parte superiore della bottiglia in plastica capovolta e larga a sufficienza per non fondere a contatto con il calore (fig.1).

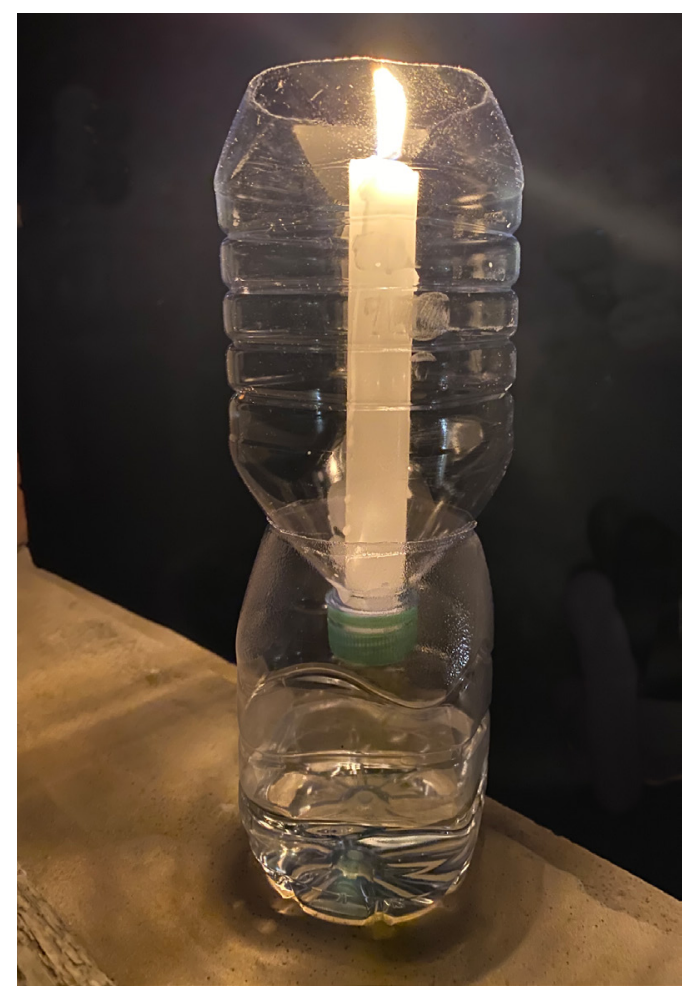

Figura 1.

La soluzione descritta rispetta i processi, i princìpi e i valori che abbiamo considerato come identificativi della cultura del progetto (§ 1), ma ciò è sufficiente per produrre design? È design quando si opera seguendo un processo progettuale come potrebbe fare un designer per sopperire a una mancanza 
Vol 21, No 24 (October 2020) • DOI: 10.12977/ocula2020-39

Michela Deni | Introduzione. La cultura del progetto, quando è design

e per migliorare la qualità della vita? Il risultato ottenuto è design se è stato prodotto da un designer? Se il processo progettuale e il risultato "individuale" e "puntuale" ottenuto nell'esempio descritto vengono prodotti su larga scala, perfezionati attraverso la cura di dettagli che ne garantiscano una qualità estetica (anche del riuso) e commercializzati, diventano design, alla stregua del design industriale? Il fatto che si possa beneficiare di una soluzione collettivamente, che tale soluzione sia replicabile, che sia firmata o brevettata, $o$ meno, garantisce le condizioni di possibilità che ci indicano quando è design? Certamente la soluzione adottata non è neanche artigianato poiché, anche se siamo di fronte a un oggetto unico (ma replicabile), non è stato prodotto grazie a un particolare saper-fare tecnico.

Si tratta invece di un'operazione di riciclo, di una trasformazione mereologica (relazione tra le parti che compongono un oggetto), di una composizione di elementi e oggetti (acqua per stabilizzare la base, bottiglia e candela) che non subiscono necessariamente una decontestualizzazione poiché la bottiglia d'acqua sta sul tavolo così come la candela all'interno di questo nuovo paralume.

\subsection{Trasformazioni e innovazioni}

L'esempio della bottiglia trasformata in portacandele con paralume è il risultato di un processo che rispetta metodi e valori della cultura del progetto poiché portano a una soluzione semplice che risolve una mancanza in modo creativo. Questa soluzione rispetta l'etica, la sostenibilità e la qualità della vita, come l'intimità di un punto luce che non prevale sulla luminosità di un cielo stellato e non ha un impatto sulle risorse energetiche limitate. Tuttavia non è possibile affermare che il risultato sia "design", poiché ci si avvicina al design "quando" numerosi altri criteri completano il processo progettuale che non è fatto soltanto di metodo ma anche di un risultato a vocazione collettiva che, nel migliore dei casi, trasforma da quel momento in poi la quotidianità in modo innovativo e radicale (cfr. Manzini 2018): come nel nostro esempio, si può cominciare da trasformazioni quotidiane circoscritte come la diminuzione del consumo elettrico nel rispetto dell'ecologia luminosa, il riuso, il riciclo e l'economicità. Un processo progettuale può dar luogo allora a un'innovazione incrementale che modifica superficialmente le nostre abitudini e ha un impatto limitato (l'auto elettrica ad esempio non modifica il nostro stile di vita). Nel lungo termine l'innovazione incrementale non modifica "l'abitabilità del mondo" poiché risponde alle stesse logiche che hanno provocato il problema (traffico, inquinamento) diventando soltanto una trasformazione inerziale che non comporta grande impatto né vantaggi per la società. Ci sono invece progetti che favoriscono un'innovazione radicale (cfr. Manzini 2018) attraverso soluzioni che danno adito a un cambiamento più profondo nello stile di vita (un servizio di bike sharing, un'organizzazione urbana che privilegi l'efficacia dei trasporti pubblici rispetto ai mezzi personali, ecc.).

Vorremmo allora poter dire che è design quando tramite un progetto si favorisce un'innovazione radicale che può avvenire quando anche la cultura 
Vol 21, No 24 (October 2020) • DOI: 10.12977/ocula2020-39

Michela Deni | Introduzione. La cultura del progetto, quando è design

del progetto muta trasformandosi in culture del progetto ${ }^{14}$ che rendono possibile tale mutamento. Per Manzini, in particolare, i criteri che caratterizzano la "nuova" cultura del progetto sono quelli propri al social design che integrano i servizi collaborativi affiancandoli a una produzione distribuita e aperta. Il design partecipativo e il co-design forniscono strumenti "di supporto alla progettazione" che "permettono di arrivare a risultati condivisi" migliorando le «capacità progettuali di tutte le persone coinvolte» (Manzini 2018: 182). L'obiettivo è di rendere il mondo «più prossimo a come si vorrebbe che fosse» afferma Manzini:

Farlo implica dunque: la valutazione critica dello stato di cose, l'immaginazione di come si vorrebbe che fosse, la disponibilità del sistema di relazioni e degli strumenti necessari per mettere in atto la sua trasformazione. E questo sia sul piano del suo funzionamento pratico (problem solving) che su quello del suo significato (sense making). (Manzini 2018: 60-61)

Dove per noi, da semiotici, epistemologicamente ma anche metodologicamente la questione del senso deve precedere tutte le altre: ciò vale per chi progetta e per chi usa e vive ciò che è stato progettato.

\section{Conclusioni}

Per concludere, possiamo avventurarci in una prima risposta affermando che è social design quando si produce un'innovazione sociale radicale. Tuttavia, non abbiamo una risposta estensiva per indicare quando è design in tutti i settori di questo campo. Le autrici e gli autori dei saggi presenti in questo numero di Ocula propongono però delle risposte che problematizzano e allo stesso tempo chiariscono il campo del design a cui si dedicano.

Andando per ordine, il primo articolo di Alessandro Zinna ha vocazione introduttiva poiché parte dalle conseguenze del "quando" attraverso un approccio archeologico e epistemologico del design, identificando le condizioni

14 Sulle pluralità delle culture del progetto rimandiamo alla presentazione del convegno Design Culture(s) Cumulus 2021: "The working title of the Conference is "Design Culture(s)" and it is aimed to map the most advanced research and education in design, by reflecting on the idea of design as a form of culture, while drawing its field and opening to new directions. Design Culture(s) is investigating not simply on the concept of culture as a monolithic expression of a specific knowledge that reflects on itself, but as the product of an investigation that is open to many different "Cultures" which are emerging and revolving around it in society, in the places and in history. We live in an increasingly complex society, which is crossed by new social, cultural and economic challenges and at the same time it is more global and multicultural than ever, more and more enriched by new languages and the flows of people. As a consequence, design is evolving as a wideopen field with many new applications and cultural meanings.Design as expression of Culture(s) is taking into consideration the cultures we are inheriting from the past and which should be granted for the benefit of future generations, so intertwining past and future, tangible and intangible, innovation and tradition» <https://cumulusroma2020. org/?fbclid=IwAR3reYseFw2GCdjLnpFDA6uiid5ViY3JP6VXuegfwregKzbJEsw82l1G8Ic $\geq$ (pagina consultata il 10/09/2020). 
Vol 21, No 24 (October 2020) • DOI: 10.12977/ocula2020-39

Michela Deni | Introduzione. La cultura del progetto, quando è design

di possibilità dell'emergere di invenzioni innovative, mostrando come queste condizioni mutano con il tempo dalla preistoria all'Antropocene. È necessario distinguere il design dalla progettualità e tale è il compito ambizioso che si dà Salvatore Zingale proponendo al lettore una logica semiotica della progettualità. Inversamente Imma Forino parte dall'oggetto di design concreto (la scrivania) che diventa un dispositif strategico per costruire ambienti come gli uffici e, allo stesso tempo, regolare pratiche d'uso. L'osservazione del design ha bisogno di metodi e proprio su tale aspetto si concentra la riflessione di Francesco Galofaro sull'etnosemiotica che ci permette di osservare gli oggetti nel contesto della vita quotidiana insieme agli attori che popolano quel contesto. Con l'articolo di Valeria Bucchetti si passa a un altro settore del design per interrogarsi sulle condizioni che permettono di definirlo, lo scopo è di identificare quando è design della comunicazione. Ma è design, in ogni campo del design, quando si agisce all'interno di un'ideologia del design e questa è la proposta di Daniele Barbieri che lo dimostra ripercorrendo le origini della disciplina. Con Marine Royer il design è un campo di attività in piena espansione, con attenzione alla centralità dei metodi e agli attori che l'autrice descrive a partire da un progetto di social design. È ancora social design quando, come fa Béatrice Gisclard, si mettono insieme politiche pubbliche e abitanti di un territorio per gestire i rischi di catastrofi naturali. Nell'ultimo articolo Yves Voglaire ci indica le competenze proprie a un designer poiché, anche se i soggetti coinvolti nel design e nel co-design sono sempre più numerosi, quando è design è necessario un designer.

Il numero 24 di Ocula si chiude con Post-it, la conclusione di Dario Mangano che prende a metafora del design la nascita del celebre foglietto giallo adesivo: il post-it è infatti il risultato di una trasformazione semiotica, dovuta a un cambiamento di punto di vista che ha permesso di ripensare relazioni (tra dettagli, soggetti e pratiche). Quando è design non è solo risolvere problemi ma saperli vedere dove nessuno li riconosce.

\section{Bibliografia}

Alessi, Chiara,

2016 Design senza designer, Bari, Laterza.

Anceschi, Giovanni

2020 Tomás Maldonado intellettuale politecnico, Milano, il verri.

Ceri, Paolo (ed.)

2014 Luciano Gallino, l’impresa responsabile. Un'intervista su Adriano Olivetti, Torino, Einaudi.

Deganello, Paolo,

2019 Design politico, Milano, Altra economia.

Deni, Michela

2016 "Conception et création", in Wikicreation (pp. 1-20). Institut ACTE UMR 8218 (Univ. Paris 1 Sorbonne, CNRS) / LabEx CAP, <https://wikicreation.fr/ wp-content/uploads/2016/o6/Conception-and-Creation.pdf>. 
Vol 21, No 24 (October 2020) • DOI: 10.12977/ocula2020-39

Michela Deni | Introduzione. La cultura del progetto, quando è design

2018 "L’intégration de l'usager dans le design social, Stratégies d'appropriation», in Basso Fossali e Le Guern (eds), L'appropriation. L'interprétation de l'altérité et l'inscription du soi, (p. 185-198), Limoges, Lambert-Lucas.

2019 "Des sciences du design à la science du design", in Fontanille e Zinna (eds), Le dialogue entre la sémiotique structurale et les sciences. Hommage à Algirdas Julien Greimas, Langages, ${ }^{\circ}$ 213, p. 93-103.

2020 "La construction de l'actant collectif par le design", in Basso Fossali (ed.) Créativité sémiotique et institutions du sens. Dans la dialectique entre l'individuel et le collectif, Limoges, Lambert-Lucas.

Deni, Michela; Catoir-Brisson, Marie-Julie (eds)

2019 La place de l'usager en design, Ocula n. 20, 10/ 2019 | ISSN 1724-7810 | DOI: $<0.12977 /$ ocula2019-1>, <www.ocula.it>

Findeli, Alain

2005 La recherche-projet: une méthode pour la recherche en design, <http:// projekt.unimes.fr/files/2014/04/Findeli.2005.Recherche-projet.pdf >.

Floch, Jean-Marie,

1995 Identités visuelles, Paris, Puf.

Goodman, Nelson

1968 Languages of Art, Indianapolis, Bobbs-Merril (tr. it. I linguaggi dell'arte, Milano, Il Saggiatore 2008).

Julier, Guy; Munch, Anders .V.

2019 "Introducing Design Culture", in G. Julier, A.V. Munch, M.N. Folkmann, H. Jensen \& N.P. Skou (Eds.), Design Culture: Objects and Approaches, London: Bloomsbury Visual Arts, (pp. 1-15), <http://dx.doi.org/10.5040/9781474289 856.0005>.

Lévi-Strauss, Claude

1962 La pensée sauvage, Paris, Plon.

Maldonado, Tomás

1970 La speranza progettuale, Torino, Einaudi.

Manzini, Ezio,

2015 Design, when everybody designs: an introduction to design for social innovation, Cambridge (Mass.), TheMIT Press.

2018 Politiche del quotidiano, Roma, Edizioni di comunità.

Munari, Bruno

1966 Arte come mestiere, Bari, Laterza.

1971 Artista e designer, Bari, Laterza.

1981 Da cosa nasce cosa, Bari, Laterza.

Papanek, Victor

1971 Design for the Real World: Human Ecology and Social Change, New York, Pantheon Books.

Semprini, Andrea

2005 La marque, une puissance fragile, Paris, Vuibert (tr. it. La marca postmoderna, Milano, FrancoAngeli, 2006). 


\section{Pcula ${ }^{24}$}

Vol 21, No 24 (October 2020) • DOI: 10.12977/ocula2020-39

Michela Deni | Introduzione. La cultura del progetto, quando è design

Simon, Herbert A.

1969 The sciences of the artificial, Cambridge (Mass.), MIT Press.

Michela Deni è Professore ordinario di Semiotica e design, direttrice del laboratorio di ricerca PROJEKT (Design e innovazione sociale) e corresponsabile della laurea magistrale Master DIS (Design Innovation et Société, <https://dis.unimes.fr >) dell'Università di Nîmes. Michela Deni insegna anche Semiotica testuale all’Università Toulouse 2 Jean Jaurès (Département Sciences du langage). Dal 1995 al 2014 ha insegnato Semiotica e Comunicazione all'ISIA di Firenze. È autrice di numerosi saggi e articoli sulla semiotica del design e del progetto: <http://projekt.unimes.fr/fr/membres/michela-deni/>. 